\title{
EL PROBLEMA DE LA REPRESENTACIÓN EN LAS BELLAS ARTES A LA LUZ DE LA HISTORIA CULTURAL
}

\section{THE PROBLEM OF THE REPRESENTATION IN THE FINE ARTS IN THE LIGHT OF THE CULTURAL HISTORY}

\begin{abstract}
María Magdalena Ziegler Delgado ${ }^{1}$. Universidad Metropolitana. Caracas, Venezuela. mziegler@unimet.edu.ve
\end{abstract}

\section{RESUMEN}

El proceso de enseñanza-aprendizaje de la historia del arte tiende a potenciarse al concebirse desde la metodología de la historia cultural. Esto en virtud de que la historia cultural nos exige ver a la obra de arte no sólo como un hecho artístico sino también como un hecho histórico y un hecho estético. En otras palabras, como un hecho cultural. Tradicionalmente, sin embargo, la enseñanza de la historia del arte se ha circunscrito en lo concerniente a las cualidades de hecho artístico de su objeto de estudio. Con ello, únicamente se limitan las posibilidades de comprensión laprendizaje de la historia del arte. Resulta pertinente entonces evaluar cómo la historia cultural colabora con la historia del arte en la comprensión de los problemas de la representación en las Bellas Artes, fundamentalmente la pintura. Nos proponemos así establecer a la historia cultural como un lente de aumento de los factores que han incidido en la llamada «experiencia del arte» correspondiente a cada obra, a partir de la aplicación de la metodología de estudio histórico-cultural que refina la información inherente a cada obra.

PALABRAS CLAVE: Historia del Arte, Educación, Bellas Artes, creatividad, resolución de problemas.

\section{ABSTRACT}

The teaching-learning process of art history tends to be enhanced when conceived from the methodology of cultural history. This is because cultural history requires us to see the work of art not only as an artistic fact but also as a historical fact and an aesthetic fact. In other words, as a cultural fact. Traditionally, however, the teaching of art history has been circumscribed as regards the qualities of artistic fact of its object of study. With this, only the possibilities of comprehension/learning of the history of art are limited. It is therefore pertinent to evaluate how cultural history collaborates with the history of art in the understanding of the problems of representation in the Fine Arts, mainly painting. We propose thus to establish cultural history as a lens of increase of the factors that have influenced the so-called "art experience» corresponding to each work, from the application of the methodology of historical-cultural study that refines the inherent information to each work.

\footnotetext{
${ }^{1}$ María Magdalena Ziegler Delgado: Consultora en Experiencia eLearning. Académica en el área de Historia del Arte y la Cultura. Asesora SeHablaArte.com.
} 
KEY WORDS: History of Art, education, Fine Arts, creativity, problem solving.

\section{Cómo citar el artículo:}

Ziegler Delgado, M. M. (2016). El problema de la representación en las Bellas Artes a la luz de la historia cultural. Revista de Ciencias de la Comunicación e Información, 21(2), 1-20.

doi: http://doi.org/10.35742/rcci.2016.21(2).1-20

\section{INTRODUCCIÓN}

El proceso de enseñanza-aprendizaje de la historia del arte tiende a potenciarse al concebirse desde la metodología de la historia cultural. Esto en virtud de que la historia cultural nos exige ver a la obra de arte no sólo como un hecho artístico sino también como un hecho histórico y un hecho estético. En otras palabras, como un hecho cultural. Tradicionalmente, sin embargo, la enseñanza de la historia del arte se ha circunscrito en lo concerniente a las cualidades de hecho artístico de su objeto de estudio. Con ello, únicamente se limitan las posibilidades de comprensión laprendizaje de la historia del arte.

Desde la historia cultural, en cambio, se debe tener siempre presente que la apariencia final de una obra de arte es el resultado de un proceso (más o menos complejo) de solución a un problema propio de un contexto. $\mathrm{Y}$, en no pocas ocasiones, la obra en sí es el único vestigio que nos queda del problema, cuando no, a veces, incluso encierra el enunciado del mismo. Así pues, el proceso de enseñanza de la historia del arte debería apuntar no a construir identidades estilísticas, sino la enunciar ese problema, rastrear el camino hacia la solución y, por supuesto, analizar críticamente «la mejor solución propuesta» por el artista para ese problema encarnado en la obra misma, considerando la experiencia estética original.

Resulta pertinente entonces evaluar cómo la historia cultural colabora con la historia del arte en la comprensión de los problemas de la representación en las Bellas Artes, fundamentalmente la pintura. Nos proponemos así establecer a la historia cultural como un lente de aumento de los factores que han incidido en la llamada «experiencia del arte» correspondiente a cada obra, a partir de la aplicación de la metodología de estudio histórico-cultural que refina la información inherente a cada obra.

En la aplicación de la metodología de la historia cultural a la historia del arte, se asume a ésta última como un variadísimo abanico de experiencias que se traducen en experiencias creativas o creadoras, experiencias estéticas y, por supuesto, obras de arte que encierran lo que esa experiencia ha sido en su tiempo y en el marco de su sociedad.

\section{MARCO TEÓRICO}

Si entendemos el pasado como un enorme paisaje que los historiadores exploran, entonces, en el marco de la historia cultural, los historiadores deben concebirse 
como exploradores muy curiosos, para quienes cada pequeño guijarro entraña un notable y rico significado en tal paisaje. Es así que todo comienza. Muy influida por la Antropología de los años 60 y 70 del siglo pasado y siguiendo la ruta de la historia social que se construía desde la década de 1950, precedida, por supuesto, por el caro esfuerzo de la Escuela de los Anales, la historia cultural ha llegado en los últimos 20 años a una especie de momentum gloriæ, convirtiéndose en una de las metodologías de estudio histórico más difundidas y adoptadas a nivel mundial.

Más allá del camino que señalaron célebres miembros de la Escuela de los Annales, como Lucien Febvre, Fernand Braudel, Pierre Nora, Jacques Le Goff y Roger Chartier, entre muchos otros, deseamos destacar el influjo del intercambio interdisciplinar que se hace claro y evidente en la obra desarrollada por Clifford Geertz, quizás el primer antropólogo que comprendió las posibilidades de los estudios históricos concebidos desde la cultura. Sus ideas tendrán una enorme influencia en los historiadores y abrirán posibilidades que refrescarán el estudio del pasado.

Geertz (2005) vio a la humanidad existiendo en medio de redes de significados que podían ser analizadas. En otras palabras, la cultura era un documento que podía ser leído a partir de su significado simbólico en lugar de una suerte de poder con gran influencia en acciones o eventos. La cultura sería así un sistema de signos y símbolos que podría ser descrito y comprendido. Esta aproximación de Geertz (2005) a la cultura dio el impulso definitivo -y he aquí su gran importancia para la historia cultural- a nuevos tipos de fuentes para quienes estaban acostumbrados a usar casi con exclusividad las tradicionales fuentes textuales.

Ahora la literatura popular, pinturas y esculturas, materiales diversos de la vida cotidiana como los utensilios de cocina o la vestimenta, así como los rituales religiosos, comportamientos y prácticas sociales diversas eran analizados como los tradicionales textos, es decir, como evidencias de la cultura que les produjo y como elementos activos dentro de una entramada red de significados. Para desentrañar y comprender la imbricación de tales significados, Geertz (2005) propuso lo que llamó «descripción gruesa» (thick description). Ésta exige entendimiento de las estructuras conceptuales y del universo imaginativo en el cual viven los individuos para llegar más allá de la superficie de las acciones, decisiones y comportamientos involucrados. Zygmunt Bauman (2002), por su parte, expondría posteriormente que toda cultura constituye un entramado de valores, que estos podrían ser compartidos por grupos más o menos grandes y que terminan por convertirse en categorías que hacen de la vida algo comprensible, inteligible.

Es así que nutrida de muchos logros y propuestas, la historia cultural desarrollada a partir de los años 70 del siglo pasado rechaza, a fin de cuentas, la comprensión de la historia basada en las interrogantes ¿qué pasó? y ¿por qué?, reemplazándolas con el hurgar en los distintos sistemas de pensamiento y conocimiento a través del tiempo. El foco del análisis histórico queda, de este modo, ampliado y profundizado. Desde las primeras acciones renovadoras de la Escuela de los Annales en las primeras décadas del siglo $\mathrm{XX}$, era posible concebir una historia alejada de sus funciones y objetos de estudio tradicionales. Pero el impulso que Geertz dio a la 
historia cultural fue decididamente re-ordenador. Y la solidez que los estudiosos de la cultura y su dinámica añadieron ha sido determinante.

No obstante, al ser una metodología de aproximación a la comprensión del pasado, debe evitarse asumir "historia cultural» como un sustantivo. De hacerlo, notaremos que todo puede ser calificado de «historia cultural», pero si lo asumimos como un indicativo de acción, como una metodología, entonces el asunto cambia. La historia cultural debe entenderse únicamente como un enfoque metodológico que no puede conformarse con las preguntas tradicionales ¿qué pasó? y ¿por qué?, pues busca comprender lo que las cosas querían decir en su momento a las personas de su tiempo, bien individualidades, grupos o sociedades enteras. De acuerdo con esto, según lo entendemos, la historia cultural es, por un lado, el análisis de la importancia de los acontecimientos en el pasado a aquellos que los vivieron como experiencias y, por extensión, cómo estos significados cambian en el tiempo de abrirse el interés en ello.

Por otro lado, al asumirlo de ese modo, se asume también que los seres humanos que viven en una misma comunidad tendrían, al menos en cierto nivel, creencias, valores y concepciones compartidas que darían forma a sus experiencias y sentido a sus acciones. La historia cultural se ocupa así de los significados de todo ello actuando en conjunto, en una red riquísima de la cual interesan no solo los significados simbólicos sino los patrones subyacentes de creencias y comportamientos que definirían a los grupos y las comunidades. Toda la historia que se ocupa de esto es historia cultural. Cualquier historia que lo evite no es historia cultural.

En este marco, la historia del arte, la razón de este estudio, ha pavimentado su propio camino con metodologías variadas de aproximación a su objeto de estudio: la obra de arte. Se ha debatido entre lo formal de la obra de arte hasta lo filosófico en ella, pasando por las experiencias creadora y estética como puntos de interés. En cualquier caso, las propuestas son múltiples y han terminado alimentándose entre sí. Sin embargo, esta hermosa disciplina corre siempre el riesgo de que la visión que el historiador imprima en ella sea percibida como demasiado parcial e incluso, superficial.

A pesar de estos riesgos ineludibles, la historia del arte ha demostrado que el hombre "puede resultar incomprensible sin el hecho estético, que no es sólo el resultado de un grupo social determinado, sino agente de ese mismo grupo" (Fernández Arenas 1982, p. 20). Por ello, aunque es posible hablar de múltiples objetivos factibles para la historia del arte, su objeto de estudio es sólo uno: la obra de arte.

Empero, si convenimos que una obra de arte es "un producto original elaborado por el hombre artificialmente con la intención de comunicar algo" (Fernández Arenas 1982, p. 27), entonces la historia del arte no puede eliminar al ser humano de la ecuación creativa, pero tampoco puede apartarse de los contextos y las circunstancias de la experiencia creadora y de la experiencia estética. En consecuencia, en una fórmula bastante más compleja, la historia del arte debe mirar con atención también las características de la actividad cultural en la cual se produce 
la obra de arte y para la cual ésta es creada. Finalmente, al agregar la variable «tiempo» en todo el asunto, la historia del arte, es pues, también historia.

La historia del arte bien puede ser concebida entonces desde la perspectiva de la historia cultural, desarrollándose a partir de las herramientas que le son propias, pero sirviéndose del incremento interpretativo de esta última. Sin embargo, si consideramos a las obras de arte no como un mero "reflejo" de la realidad o de un contexto histórico determinado, estaremos perdiendo prácticamente toda la riqueza que las obras encierran. Las obras de arte han de asumirse como una manifestación de la sociedad y del artista, de la mentalidad y el andamiaje cultural que ambos comparten. Más aun, toda obra de arte se enriquece en su consideración como un hecho histórico, como un hecho estético y como un hecho artístico, de manera sincrónica y complementaria, cuyas lecturas deben ir de la mano cuando hacemos historia del arte.

Ernst H. Gombrich (1985a), al señalar a las obras de arte como parte de un componente funcional en la sociedad, sin el cual, su razón de ser estaría más que ausente, comparó a la obra de arte con un "caballito de juguete" (hobby horse). Al hacerlo, Gombrich buscaba colocar el acento en cómo la obra de arte es sobre todo una interpretación de la realidad del mismo modo que un caballito de juguete lo es de un caballo. En otras palabras, las obras de arte no pueden ser asumidas exclusivamente como realistas o no (en virtud de su "parecido" con la realidad), pues son, por sobre todas las cosas, interpretaciones $y$, en ese sentido, estarían íntimamente vinculadas con esa realidad que interpretan a través de la intención, propósito y función que les incumbe. Sin más, son un hecho cultural y no sólo artístico.

En este sentido, es oportuno destacar que, como bien expuso Pierre Francastel (1988a), toda sociedad se recrea en un imaginario dado que ella misma fabrica y "vive dentro de un cuadro facticio, sistemáticamente elaborado y representativo de los valores esenciales a los cuales ella se apega, sobre los cuales funda su duración" (p. 296). Consecuentemente, las obras de arte serían una interpretación material de ese vivir en un entramado que en ocasiones se concreta y, en otras, se diluye; que a veces responde a una tradición y, sin embargo, puede detonar grandes cambios inesperadamente aunque no siempre radicalmente.

Veamos más de cerca todo este proceso de análisis histórico desde la historia cultural y para la historia del arte. Por un lado, Gombrich ha expresado que "la obra de arte tiene carácter de un sueño compartido" (1985b, p. 31). Si aceptamos esto, podemos afirmar también que al definir elementos de la obra de arte devendríamos en un conocimiento mayor de la cultura en la cual está inserta, de la cual es (o ha sido) parte. Más aun, "antes de preguntarnos qué expresan [las obras], debemos saber a qué marco institucional estaban destinadas" (1985b, p. 91). Dicho de otro modo, toda obra de arte tiene un destino institucional, sea éste la familia o el palacio de gobierno. Determinarlo culturalmente es enriquecer el significado de la obra, pero no artificialmente, sino desde su propia esencia. Quizás un término más apropiado sería ya no «enriquecer» sino «desvelar».

Por su parte, Francastel ha indicado que el arte "permite no sólo anotar y comunicar representaciones adquiridas, sino también descubrir nuevas. No es sólo 
comunicación sino institución. No es lenguaje sino sistema de significación" (1988a, 15). No viene esto si no a refrendar lo que ya hemos citado de Gombrich, pero lo hace enfatizando lo que luego Geertz va a desarrollar mejor desde el punto de vista de la antropología y que mencionábamos párrafos atrás. Ese sistema de significación del que habla Francastel, devendría en la idea de Geertz (2005) de los valores culturales, asumidos generalmente como una guía de comportamiento y/o actitud antes ciertas situaciones y que mantendría a los individuos dentro de una cierta «normalidad» (existente o anhelada).

El arte sería, en suma, una suerte de toma de conciencia. Los artistas, en este marco, son los que elaboran la síntesis visual de la realidad, de aquello que luce efímero y que se anhela como permanente, de aquello que se anhela instituir (o destruir). Con una sensibilidad personal, los artistas, plantean la visión de un universo particular (su contexto), pero pueden llegar a dirigirlo a la posibilidad de aprehenderse desde lo universal. Es claro que el dinamismo natural de las artes podría hacernos pensar que las visiones que genera no pueden ser estáticas. De hecho, no lo son, a pesar de lo que las apariencias puedan decirnos. Así como los valores culturales son, para Geertz (2005), dinámicos, pues mudan sus límites y contenidos con la sociedad misma, aun cuando una sociedad actúe conscientemente a favor de la preservación de ciertos valores, también el arte se mueve con los valores culturales. A ratos enfrentándolos, a ratos refrendándolos.

La historia del arte no puede estar ajena a tales cambios y explicarlos exclusivamente a partir de modificaciones en el gusto, las técnicas o movimientos estilísticos. Por el contrario debe servirse de los cambios culturales, está en la obligación de comprenderlos, de esbozar al menos una mentalidad que crea en un momento determinado en función de unas exigencias (de diversa índole), impulsada por una intención (personal del artista o de un grupo social) y determinada en su propósito final.

Son los artistas los que ponen en práctica métodos creativos para organizar la forma como el mundo es percibido dentro de su propia cultura. Recurriendo nuevamente a Gombrich, nos encontramos con que -en una interesante conversación con Didier Eribon- expone que "en el arte no es posible la comunicación sin un lenguaje común" (1993, p. 91), por lo que no sería posible comprender realmente a las obras de arte si no se tienen presentes las expectativas que la sociedad pudiera tener sobre ellas, sobre su complexión, apariencia y funciones. Así pues, es clave considerar aquello que un artista haya podido disponer para asegurar una comunicación óptima a través de sus obras.

Pero comunicar en el sentido en el que el arte comunica, no puede reducirse a establecer un estilo para las obras. Por mucho tiempo los historiadores consideraron el estilo de una obra de arte una materia esencial de investigación. Le interesaban las correspondencias internas de un estilo, su duración o su vida, así como los problemas de su formación y cambio. Meyer Shapiro, en un artículo publicado en la revista Anthropology Today, titulado "Style" (en Preziozi, 1998), por ejemplo, llegó a considerar que un estilo artístico era un sistema de formas con una cualidad y una expresión significativa a través del cual la personalidad del artista y la amplia visión de un grupo se hacían visibles. 
Svetlana Alpers (en Lang, 1987), por otro lado, ha manifestado su perturbación ante el hecho de que el historiador del arte designe un estilo a una obra, pues esto se termina asumiendo como si la obra en sí poseyera al estilo. Tanto es así que no tiene reparos en afirmar que "a menudo el valor de un objeto depende de que le sea asignada una identidad estilística” (Alpers en Lang, 1987, p. 139). En sintonía con esto, ya en 1955, Gombrich (1985b) había advertido sobre las limitaciones del análisis formal, alertando que toda terminología estilística está cargada de valores que no necesariamente se corresponden con los propios de las obras. Esto es indiscutible, pues la mayor parte (por no decir todas) las etiquetas que se emplean para designar estilos tuvieron originalmente una carga peyorativa abierta. Pero incluso, en el caso que no haya sido así, las etiquetas las coloca el historiador del arte desde el presente, no son legados del propio tiempo de las obras de modo general. Emplearlas exclusivamente sería, pues, una suerte de perversión metodológica de la historia del arte.

Con gran honestidad, Gombrich expuso entonces que cada historiador y crítico, guiado por el empeño de la clasificación estilística, siempre se hallará en la situación de sacrificar alguna de sus exigencias o de subordinar la mayor parte de ellas a fin de que la clasificación no naufrague. "El riesgo de todos los 'ismos' es volverse un postulado, y el de muchos escritos sobre arte singularizar tanto un aspecto que llegan a olvidar los demás" (Gombrich, 1985b, p. 179). Esto, evidentemente, genera un problema que es muchísimo más difícil de resolver que la aplicación de la manida metodología de asignación y descripción de identidades estilísticas.

Pero si nos deshacemos de la terminología estilística tradicional de la historia del arte, si desestimamos la importancia que a lo largo de los años se le ha dado a construir una identidad estilística para cada obra de arte (y grupos de ellas), entonces ¿qué nos queda por hacer desde la historia del arte? Todo. Nos queda todo por hacer. Si bien es verdad que una obra de arte es nula sin lo formal, también es verdad que lo formal no se agota en lo estilístico.

Por lo tanto, lo formal debería dar paso a la construcción de un conocimiento más complejo que el resultante de una serie de características reunidas bajo una etiqueta (académico, realista, naturalista, barroco, etc.). Si partimos del supuesto de que lo formal en el arte tiene una carga de información particular que no se limita a ella misma, sino que trasciende los límites de lo sensible y que se vincula con valores de diversa índole, tendríamos un motivo de peso suficiente para evitar que el análisis formal desemboque únicamente en el otorgamiento de insignias que acrediten a una obra como poseedora de uno u otro estilo.

¿Cómo proceder entonces? El historiador del arte debe tener siempre presente que la apariencia final de una obra de arte es el resultado de un proceso (más o menos complejo) de solución a un problema. En este sentido, lo formal, esto es, el uso de los elementos de expresión visual (forma, color, luz, composición, etc.), no es sino el registro que permanece (por siglos, años o minutos) del uso de herramientas diversas procurando la mejor solución posible a un problema. En no pocas ocasiones, la obra en sí es el único vestigio que nos queda del problema, cuando no, a veces, encierra el enunciado del mismo. Así pues, la labor del historiador del arte no ha de ser la de construir identidades estilísticas, sino la enunciar el problema, 
rastrear el camino hacia la solución -con sus altos y bajos- y, por supuesto, analizar críticamente «la mejor solución propuesta» para ese problema encarnado en la obra misma.

\section{METODOLOGÍA}

Desde la perspectiva de la historia cultural, solucionar cualquier problema demanda un cierto orden de elementos, pero también demanda una definición clara del problema y una diáfana conciencia de las posibilidades de las herramientas con las que se cuenta para resolverlo. Desde la perspectiva de la historia del arte, todo artista, en cualquier tiempo o contexto, se ha enfrentado con problemas de distinta índole, bien artísticos, bien políticos, bien religiosos o sociales, incluso económicos y hasta de orden psicológico. Con ello, la historia del arte busca comprender interpretaciones culturales y no simplemente creaciones estilísticas.

Sin embargo, debe decirse que "cuando en arte se habla de resolver problemas hay que poner cuidado en evitar la impresión de que el arte es una forma superior de crucigrama" (Gombrich 1985b, p. 180). Hay una razón para esto y es que quien se dedica a resolver un crucigrama o una sopa de letras lo hace a sabiendas de que existe una solución óptima. "En el arte, tal garantía no existe" (Ibídem). Así pues, lejano a cualquier garantía de que el problema detectado o planteado posee una solución óptima y de que ésta estará a su alcance, el artista traduce su empeño en experimentación, estudio y búsqueda, sin que necesariamente estas tres acciones tengan ese orden o ningún otro en específico; ni siquiera podrían llegar a ser necesarias las tres.

A partir de lo anterior, se abre delante del historiador del arte un campo enormemente atractivo para su estudio, aunque también complejo. Estudiar una obra de arte sin tener los problemas del estilo como un fin sino como un medio, uno de tantos que deben considerarse, es estudiar a la obra de arte desde una perspectiva cultural amplia, histórica y, por supuesto, artística. Sería hacer historia del arte como historia cultural. Consideremos además que el arte es una interpretación material del vivir en el entramado cultural de una sociedad. Francastel señala que los artistas no son únicamente creadores de objetos bellos sino de "esquemas de pensamiento", por lo que para él "existe, en una palabra, un pensamiento plástico lo mismo que existe un pensamiento matemático 0 un pensamiento político" (1988b, p. 13).

De esta manera, debemos deshacernos también de la idea de que el arte es un reflejo de la sociedad. En modo alguno, el arte podría ser considerado tal, ni su traducción en imágenes. Se trata más bien de "una trampa lingüística" como la llama Vicenç Furio (2000, p. 114). Si el arte no fuera sino mero reflejo de la sociedad, entonces valdría estudiar a la sociedad y ya conoceríamos todo lo que hay que saber sobre el arte. "El artista no se limita a pasearse con un espejo, a reproducir pasivamente la realidad social” (Ibídem).

Elementos extra-artísticos podrían condicionar el arte, pero esto no significa que el arte sea un reflejo de estos elementos. Metodológicamente, la historia del arte debe partir del principio de que el arte conlleva a una forma de pensamiento particular, por 
lo que, consecuentemente, no podría hablarse de equivalencias entre el arte y la política, sino de brazos comunicantes, de puentes entre el pensamiento plástico y el pensamiento político o religioso, por ejemplo.

El pensamiento plástico "es uno de los múltiples modos por los cuales el hombre influye o da forma al universo que le rodea" (Francastel, 1988b, p. 14). Reconocer que los artistas, en distintas épocas, elaboran un pensamiento plástico propio, es reconocer también que estos son capaces de elaborar una versión racionalmente organizada de su contexto, de las necesidades del mismo e incluso de los anhelos para el futuro. Aun cuando aceptemos que no todos los artistas tendrían la capacidad y/o el talento para tal cosa, es posible que estos artistas menos talentosos copiaran lo que otros ya habrían elaborado. En todo caso, esto no elimina la existencia de un pensamiento plástico en sí.

Desde este punto de vista, para la historia del arte dejan de ser los artistas seres pasivos o despreocupados artesanos que con abundantes o limitados recursos técnicos realizan obras sin más mérito que las habilidades técnicas impuestas en ellas. En los predios metodológicos de la historia cultural, para la historia del arte los artistas interpretan y lo hacen aun cuando deseen copiar literalmente la realidad. Es importante partir de ese hecho. La obra de cualquier artista nos informa sobre su modo de pensar (y el del círculo al que pertenecía). En palabras de Francastel, "la obra está en lo imaginario" (1988b, p. 29).

El historiador del arte debe entonces, a partir de las obras, desplegar ese mapa cultural que le mostrará a las propias obras como referentes. Francastel llama a la obra de arte un «lugar de enlaces» para los anhelos y la realidad. "Todo nuevo pensamiento se encarna en nuevos objetos y crea lugares imaginarios, tanto figurativos como reales" (Francastel 1988a, p. 296). Evidentemente, es posible que no siempre este mapa se despliegue claro y diáfano, porque en los momentos culturalmente más confusos, en aquellos que pudiéramos pensar que hay ausencia de valores culturales, Bauman nos advierte que, muy por el contrario, lo que tenemos frente a nosotros es una multitud de valores que se encuentran "escasamente coordinados y débilmente vinculados a toda una discordante variedad de autoridades" (2002, p. 92).

A la atención del historiador del arte queda expuesta no sólo la obra de arte y sus cualidades artísticas y estéticas, sino también e ineludiblemente el artista, su circunstancia y la institucionalidad en la cual se desenvuelve. Pero además, el historiador del arte no puede eludir al público, porque finalmente la obra de arte es creada para ser percibida, bien por una persona, bien por una gran masa homogénea o heterogénea. De manera pues que la recepción de la obra, el modo como se disfrutó en su tiempo, el rechazo que pudo haber provocado o la emoción que desató debe ser parte de las consideraciones del historiador en su estudio. De lo contrario, estaría presentando sólo una porción de la experiencia del arte.

Es justo aquí que no solo el estudio de la historia del arte, sino también su enseñanza y aprendizaje se potencia y amplían. Al desechar la lista de estilos, la enseñanza debe sumergirse en la experiencia del arte y a través de ella facilitar la comprensión del pasado. El aprendizaje se ensancha y deja muy lejos la 
memorización de nombres, fechas y adjetivos, para re-visitar la experiencia creadora y la experiencia estética de una obra de arte que se asume como un hecho cultural de gran significación.

Se tiene entonces que para existir, toda obra de arte requiere de un creador, de alguien que la conciba, que haya pensado en ella, que le haya imaginado y, por qué no, que le haya soñado. Ese creador lo conocemos como artista y es a él exclusivamente al que debemos la experiencia creadora. De esta experiencia surge la obra de arte como una realidad sensible, poseedora de cualidades artísticas y estéticas, pero también culturales y, en consecuencia, históricas. La experiencia estética, como fase final de la "experiencia del arte», queda así en manos del espectador, de aquel para quien la obra fue creada, de aquel que la percibe fortuitamente o deliberadamente, de quien se enfrenta a ella al momento de su creación o siglos después.

Son tres esferas de la experiencia del arte que se complementan y agregan comprensión a la historia del arte desde la historia cultural. En términos de la experiencia creadora, al profesor se le abrirá un campo de enormes posibilidades para señalar las etapas de los procesos creativos, la importancia del error en la búsqueda del acierto y los modos de concreción de soluciones a problemas de diversa índole. Al estudiante, los procesos creativos se le harán cercanos, se le mostrarán las dificultades de crear y las posibilidades de la creación como proceso de identificación de problemas y de generación de óptimas soluciones.

Por otra parte, la experiencia estética, tan obviada por la historia del arte tradicional, presenta la oportunidad de comprender procesos psicológicos de percepción, de persuasión, de emoción, etc. Las relaciones entre las obras de arte y los espectadores también tienen una historia, es parte de la historia misma del arte. Es enorme el vacío que existe en este sentido, porque abundan las evidencias acerca de estas relaciones, de las reacciones, de las emociones y respuestas de las personas de todo tipo respecto a las obras de arte. En el estudio de la experiencia estética podría hallarse un cúmulo de respuestas, un acervo de información que nos llevaría directamente a la propia obra y, claro está, a la experiencia creadora. Después de todo, "Ios artistas no hacen sino materializar los valores del medio en que viven" (Francastel 1988a, p. 12).

Observamos, pues, que la experiencia del arte es un universo inmenso, de matices y aristas variadas y heterogéneas que no pueden ser aprehendidas ni comprendidas a partir de las mismas herramientas. De hecho, ninguna obra de arte puede encerrar una verdad, sino muchas o incluso sólo una porción de ella. Aunque muchos continúen defendiendo la absurda idea de que el arte es un lenguaje universal, el historiador del arte debe asumir esto como una falsedad. Esto, sobre todo, porque el contexto es primordial para entender y aprehender cualquier obra de arte.

Desvincular a las obras de su contexto es mutilarla. Furió (2000, p. 83) ha dicho que es justamente el contexto lo que permite explicar las obras de arte "como una solución a ciertos problemas en determinadas circunstancias". Situar las obras en su espacio y tiempo es capital si deseamos establecer el grado de innovación, creatividad o sencillamente de complejidad técnica o capacidad expresiva. Con ello 
los estudiantes no asumirán la obra de arte como un objeto ajeno a toda influencia externa, sino como una creación generada por un proceso personal y destinada a un público en particular esperando -en muchos casos- una respuesta específica. $Y$ es que "enjuiciar la calidad de una obra sin situarla históricamente es emitir un juicio vacío" (Furió 2000, p. 83). No es posible limitarse tan sólo a una valoración artística formal que tendría que ver más con nuestros gustos que con los de aquellos que las crearon y para quienes fueron creadas. Los profesores enseñarían una ilusión y los estudiantes aprenderían un ensueño.

En el mundo de hoy, los profesionales -en todas las áreas- son considerados como solucionadores de problemas y se espera que sean capaces de encarar demandas cada vez más complejas a través de soluciones creativas, sostenibles y factibles. Valqui Vidal (2009) considera que todo profesional debería ser capaz de redefinir constantemente las habilidades necesarias para enfrentar una tarea determinada. Cuando los individuos quedan atrapados en modos tradicionales de resolución de problemas, pierden la habilidad para localizar alternativas de solución, en consecuencia, "los métodos creativos son necesarios para resolver problemas en la práctica" (Valqui Vidal, 2009, p. 1). En este sentido, "crear es una reacción en cadena; puede ser usada por todos. El inteligente piensa, proyecta, potencia imaginativamente, se plantea tareas" (Torres Soler, 2002, p. 82).

La historia del arte, tal y como la hemos presentado aquí, permite que los estudiantes renueven constantemente sus modos de aproximación a los problemas que deben afrontar. Desarrollando un pensamiento creativo robusto se accede a "un estilo de vida, una orientación de la personalidad, un modo de ver el mundo una forma de interactuar con los demás, una manera de trabajar en equipo, una forma de vivir y desarrollarse" (Valqui Vidal, 2009, p. 2). En otras palabras, la historia del arte en su enfoque cultural, en la importancia brindada a la experiencia del arte, puede llegar a convertirse en una herramienta de liberación de esquemas mentales que derrotaría las costumbres, los hábitos y las rutinas infructuosas.

La necesidad de que los más jóvenes (sobre todo los niños) puedan tener la oportunidad de explorar el mundo y sus creaciones, de descubrir en ellos nuevas ideas y modos de comprenderlo e interpretarlo, de experimentar para que los errores puedan también enseñarles, es ya hoy un asunto de primer orden en los procesos de enseñanza y aprendizaje. "Ser creativo es explorar lugares nuevos e ideas nuevas. Es desarrollar una sensibilidad a problemas de otros y problemas propios. Es usar la imaginación para producir ideas y resolver problemas" (Torres Soler, 2002, p. 84).

La historia del arte como la historia de la capacidad creativa del ser humano permite asumir a través de ella un proceso de enseñanza/aprendizaje que coloque a la creatividad como la búsqueda y solución de problemas en nuestra realidad. Porque para identificar un problema correctamente debe conocerse el entorno y la práctica se ejecuta en el conocimiento de contextos históricos. De esta manera, la creatividad se asume como "una visión problematizadora sobre la realidad que nos rodea, sobre nuestra experiencia, buscando siempre otras lecturas, otras perspectivas, nuevas respuestas ante variados y concretos problemas de la experiencia siempre multifacética" (Romero, 2002, p. 308). 
La historia del arte como historia cultural ofrece un universo de posibilidades de exploración y de enriquecimiento de la disciplina que sería imposible medir. Todo lo que se ha escrito sobre el arte debe rescatarse, debe emplearse para brindar nuevas luces, pero no debe seguirse sosteniendo que se ha dicho todo sobre una obra al desentrañar su estilo o su origen. La historia del arte debe enseñarse como la historia de la más extraordinaria capacidad del ser humano: la capacidad de crear. Sólo así el estudiante podrá desarrollar las competencias necesarias para comprender lo que él mismo podría ser capaz de crear, en tanto humano, ante ciertas circunstancias y ante determinadas exigencias.

\section{REFERENCIAS}

Bauman, Z. (2002). La cultura como praxis. Barcelona: Paidós Studio.

Fernández Arenas, J. (1982). Teoría y Metodología de la Historia del Arte, Anthropos, Barcelona.

Fernie, E. (1995). Art History and its Methods: A critical anthology. Londres: Phaidon Press.

Francastel, P. (1988a). La figura y el lugar. Caracas: LAIA-Monte Ávila.

Francastel, P. (1988b). La realidad figurativa. Barcelona: Ediciones Paidós.

Geertz, C. (2005). La interpretación de las culturas. Barcelona: Gedisa.

Gombrich, E. (1985a). Meditations on a hobby horse. London: Phaidon.

Gombrich, E. (1985b), Norma y Forma, Alianza, Madrid

Gombrich, E. (1993). Lo que nos dice la imagen (conversaciones con Didier Eribon). Bogotá: Norma.

Lang, B. (Ed.) (1987). The concept of style. Nueva York: Cornell University Press.

Preziosi, D. (Editor) (1998). The art of art history. Oxford University Press.

Romero, J. (2002). "Cambios de perspectiva: Educación artística, creatividad y arte infantil", en Arte, individuo y sociedad, № Extra 1.

Thuillier, J. (2006). Teoría general de la Historia del arte. México: Fondo de Cultura Económica.

Torres Soler, L. (2002). Un camino al éxito: la creatividad, en Ingeniería e Investigación, 50.

Valqui Vidal, R. (2009). Creatividad: conceptos, métodos y aplicaciones, en Revista Iberoamericana de Educación, 49/2. 